\title{
The Structure of Induced Diffusion Flows on a Wedge with Curved Edges
}

\author{
N.F. Dimitrieva ${ }^{1}$, Yu.D. Chashechkin ${ }^{2}$ \\ ${ }^{1}$ Institute of Hydromechanics, National Academy of Sciences of Ukraine, Kiev, Ukraine \\ e-mail: dimitrieva@list.ru \\ ${ }^{2}$ A.Yu. Ishlinsky Institute for Problems in Mechanics, Russian Academy of Sciences, \\ Moscow, Russia \\ e-mail: chakin@ipmnet.ru
}

\begin{abstract}
The mathematical model and the numerical implementation method permitting to study simultaneously all the elements of the internally multi-scale stratified flows are developed. For the first time, the induced diffusion flows on a fixed symmetrical wedge with the curved edges are calculated and visualized using the supercomputer systems. Influence both of the edge effects and the wedge lateral edge curvature upon the flow structure is shown. In a stably stratified environment, the integral force inducing self-motion of a free wedge along the neutral buoyancy horizon towards the top is formed by pressure deficit in the adjacent jet streams.
\end{abstract}

Keywords: stratified fluid, induced diffusion flows, self-motion.

DOI: 10.22449/1573-160X-2016-3-70-78

(C) 2016, N.F. Dimitrieva, Yu.D. Chashechkin

(C) 2016, Physical Oceanography

Introduction. Dissolved substances in the fluid and suspended particles under the influence of the mass forces (the gravitational or centrifugal ones) are unevenly distributed and form a stable stratification. Its density field is determined by the temperature, impurity concentration and pressure profiles in accordance with the type of the equation of state. Nonequilibrium medium with molecular flow of the stratifying components is resting only if density gradients are parallel to the gravitational vertical. The molecular flow interruption at the impermeable boundaries of arbitrary shape generates induced diffusion flows. Theoretical studies of these flows started in the early 40s of the last century [1], and a little later they began to be simulated in the laboratory [2]

Interest to the study of gradient flows is maintained due to their prevalence in the environment. Under normal conditions, the perturbations are concentrated in thin layers at the impermeable surfaces and reach storm values forming large temperature gradients in the atmosphere at steep range slopes [3] and nearby glaciers [4]. In recent years, special attention is paid to the study of the influence of induced diffusion flows on the dynamics and structure of processes in the reservoirs, lakes, seas [5] and oceans, taking into account the influence of the global rotation [6]. The complexity of the equation of state of the marine environment and the presence of an additional stratifying component with its own diffusion coefficient («differential diffusion») was considered in some studies [7].

At the end of the last century the models of the process of setting of induced diffusion flows at various obstacles (inclined and horizontal plate [8, 9], cylinder and sphere) started to be developed along with analytical studies of stationary currents $[1,2,5]$. The laboratory tests using highly sensitive shadow devices were also carried out. In the experiments except large eddies the rosettes of dissipative 
non-stationary gravity waves were observed at the obstacle poles. Removing from the obstacles, these rosettes were transformed into extended layers [10].

The integral force action of convergent flows, absent in the symmetric obstacles (sphere, cylinder or horizontal plate), assumes a finite value on the inclined plate and other bodies, non-symmetrical with respect to the line of gravity. The resulting pressure gradients are large enough and capable of inducing selfmotion of the neutral buoyancy free bodies («diffusion fish» in parlance of article [11]), which plays an important role in the dynamics of the hydrosphere. Calculation of steady flows on the flat fixed wedge is carried out in [12]. The study of formation mechanism of the propulsive forces on the bodies having various shapes is of practical interest.

In the present paper, for the first time the setting up dynamics and structure of the two-dimensional induced diffusion flows on concave or convex symmetrical wedge was calculated.

Problem formulation and its solving method. Since the velocities of the studied flows are small compared to the sound velocity, the mathematical model of the given physical processes is based on the system of differential balance equations of inhomogeneous multicomponent fluid mechanics in the Boussinesq approximation and neglection of the compressibility effects [13]. This system includes the continuity equation, the momentum balance (Navier - Stokes), the substances and closing algebraic equation of state $\rho(S(z))$ :

$$
\begin{gathered}
\rho=\rho_{0}(\exp (-z / \Lambda)+s), \\
\operatorname{div} \mathbf{v}=0, \\
\frac{\partial \mathbf{v}}{\partial t}+(\mathbf{v} \nabla) \mathbf{v}=-\frac{1}{\rho_{0}} \nabla P+v \Delta \mathbf{v}-s \mathbf{g}, \\
\frac{\partial s}{\partial t}+\mathbf{v} \nabla s=\kappa_{s} \Delta s+\frac{v_{z}}{\Lambda},
\end{gathered}
$$

where $S=S_{0}(z)+s$ is the total salinity, including the salt compression ratio with initial stationary stratification and perturbation marked $S(x, z, t), \rho_{0}$ is the density at zero level (neutral buoyancy level); $\mathbf{v}=\left(v_{x}, v_{z}\right)$ if the velocity of fluid; $P$ is the pressure excluding the hydrostatic one; $v$ and $\kappa_{s}$ are the constant coefficients of kinematic viscosity and salt diffusion; $t$ is the time; $\mathbf{g}$ is the acceleration of gravity; $\nabla$ and $\Delta$ are the operators of Hamilton and Laplace; $\Lambda=\rho /|\nabla \rho|, N=\sqrt{\mathbf{g} / \Lambda}$, $T_{b}=2 \pi / N$ are the scale, frequency and period of buoyancy; the $z$ axis is directed vertically upward. Member with the vertical component of the flow velocity in the formula (4) represents the convective transport of salinity, violating the initial stratification.

At the initial time $t=0$ in the resting continuously stratified fluid the impenetrable wedge of the selected shape is placed horizontally. On the surface of the body $\sum$ the adhesion condition for the substance velocity and impermeability is set, and at an infinite distance - the decay of all disturbances:

PHYSICAL OCEANOGRAPHY NO. 3 (2016) 


$$
\begin{gathered}
\mathbf{v},\left.s\right|_{t \leq 0}=0,\left.\quad \mathbf{v}\right|_{\Sigma}=0, \quad \mathbf{v},\left.s\right|_{x, z \rightarrow \infty}=0 \\
\left|\frac{\partial S}{\partial n}\right|_{\Sigma}=-\frac{1}{\Lambda} \frac{\partial z}{\partial n}+\left|\frac{\partial s}{\partial n}\right|_{\Sigma}=0
\end{gathered}
$$

where $n$ is the outward normal to the obstacle.

A set of dimensional parameters $\left(v=10^{-6} \mathrm{~m}^{2} / \mathrm{c}, \quad \kappa_{\mathrm{s}}=1,41 \cdot 10^{-9} \mathrm{~m}^{2} / \mathrm{s}, g_{z}=9,8\right.$ $\mathrm{m} / \mathrm{s}^{2}, N=1 \mathrm{~s}^{-1}$ )is presented in the problem. They form the characteristic time scales $t=T_{b}$, including the linear macroscales (of buoyancy $\Lambda=9,8 \mathrm{~m}$, of the obstacle size $L=0,1 \mathrm{~m})$ and microscales $\left(\delta_{N}^{v}=\sqrt{v / N}=10^{-3} \mathrm{~m} \quad\right.$ and $\left.\delta_{N}^{\kappa_{s}}=\sqrt{\kappa_{s} / N}=4 \cdot 10^{-5} \mathrm{~m}\right)$. The characteristic velocity scale is given by the ratio of the basic scales $U_{N}^{v}=\sqrt{v N}=10^{-3} \mathrm{~m} / \mathrm{s}, U_{N}^{\kappa_{s}}=\sqrt{\kappa_{s} N}=4 \cdot 10^{-5} \mathrm{~m} / \mathrm{s}$.

Macroscales $\Lambda$ and $L$ characterize the initial stratification and geometry of the current. Dissipation nature microscales $\delta_{N}^{v}$ and $\delta_{N}^{\kappa_{s}}$ determine the lateral dimensions of the fine structure components. A wide range of the values of length scales indicates the complexity of the stratified current internal structure.

Research of the linearized fundamental equations and the results of laboratory modeling showed the large-scale elements of currents (waves and eddies) to be characterized by regular perturbation components of the total solution. Fine structure elements of the flows, visualized in the whole range of parameters of the studied processes, are described by the large variety of singularly perturbed components. All the components coexist and actively interact with each other, forming a fine evolving structure [14].

Scale ratios set the traditional dimensionless complexes, such as the buoyancy scale $C=\Lambda / L$, the Reynolds number $\operatorname{Re}=|\mathbf{v}| L / v$, the Péclet number $\mathrm{Pe}=|\mathbf{v}| L / \kappa_{s}$, the Schmidt number Sc $=v / \kappa_{s}$ and the Froude number $\mathrm{Fr}=|\mathbf{v}| / N L$, where $|\mathbf{v}|$ is the flow velocity. For the solution of the problem of induced diffusion flow on a fixed obstacle it is taken to be equal to $U_{N}^{\kappa_{S}}$.

In the nonlinear formulation the system (1) - (6) provides the simultaneous study of all elements of flows within the single description in natural physical variables without additional constants and ratios. Due to multi-scale phenomena and nonlinear equations, one of the main tools for analysis of such evolutionary processes is the numerical modeling.

Computational domain sampling was carried out on the basis of the SALOME open-source generic platform (www.salome-platform.org), including blockMesh, topoSet and refineMesh generating utilities supplied by OpenFOAM open source software (www.openfoam.com), taking into consideration their individual scales. The computational domain is a rectangle with the horizontally arranged symmetrical wedge with $L=10 \mathrm{~cm}$ length and $h=2 \mathrm{~cm}$ base height. The sides of the body are arcs of circles and radii. The positions of their centers in a Cartesian coordinate system centered at the wedge top are shown in Tab. 1. 
Wedge Geometry

\begin{tabular}{c|c|c}
\hline $\begin{array}{c}\text { Coordinates of the center, } \\
\text { сM }\end{array}$ & Curvature radius, cm & $\begin{array}{c}\text { Deviation from the face } \\
\text { of the chord } \Delta_{R}, \mathrm{~cm}\end{array}$ \\
\hline$(-7.6 ; 126.7)$ & 126.9 & -0.1 \\
$(17.6 ; 125.7)$ & 125.7 & 0.1 \\
$(9.2 ; 41.4)$ & 43.2 & 0.3 \\
$(5.8 ; 126.7)$ & 10.0 & 1.4 \\
\hline
\end{tabular}

Taking into account the shapes of the body the hexahedral block-structured grids with registration of the lines on the block boundaries were reconstructed. For resolution of the fine structure components of flows to a minimum microscale $\delta_{N}^{\kappa_{S}}$ several cells of the computational grid in domains with large gradients were nested. The fulfilled test calculations on a coarser grid showed the local non-physical oscillations of the solution near the obstacle. These oscillations lead to the accumulation of errors and stop of the counting during continuous computations.

Numerical solution was based finite volume method in the open source software OpenFOAM. To take into account the stratification and diffusion effects the standard numerical model icoFoam, realizing the non-stationary flows of the homogeneous fluid, was supplemented with new variables ( $\rho$ and $s$ ) and the appropriate equations (1) and (4), as well as with new additional parameters $(N, \Lambda$, $\kappa_{s}, g$, etc. [15]).

The boundary condition for salinity perturbation (6) was implemented by the extended utility funkySetBoundaryField, which permits to set the analytical expressions for the physical variables. To conduct the further analysis applying such utilities, as vorticity, funkySetFields, probes, forces and forceCoeffs, the informative physical quantities (vorticity and its baroclinic generation, rate of dissipation of mechanical energy) lacking in the standard OpenFOAM software package, were calculated.

Results and discussion. The complex multi-scale structure of the induced diffusion flows is illustrated by the field of the horizontal component of salinity perturbation gradient (Fig. 1). Positive values of the visualized value are indicated in light gray, negative ones - in dark gray. There is the same range of value change between the isolines. The image structure is consistent with the data of shadow flow visualization in a laboratory pool [11].

Nearby the corner points of the wedge the additional fine structure elements the dissipative gravitational waves - are formed. Their maxima of the longitudinal component of salinity gradient perturbation reached $|\partial s / \partial x|_{\max }=4 \cdot 10^{-2}$ (salinity perturbations $s$ had order of $10^{-5}$ ). With the removal of obstacles, the salinity perturbation gradient sharply decreases and reaches about $10^{-6}$ at $5 \mathrm{~cm}$ distance horizontally and $0.5 \mathrm{~cm}$ vertically from the corner point. 

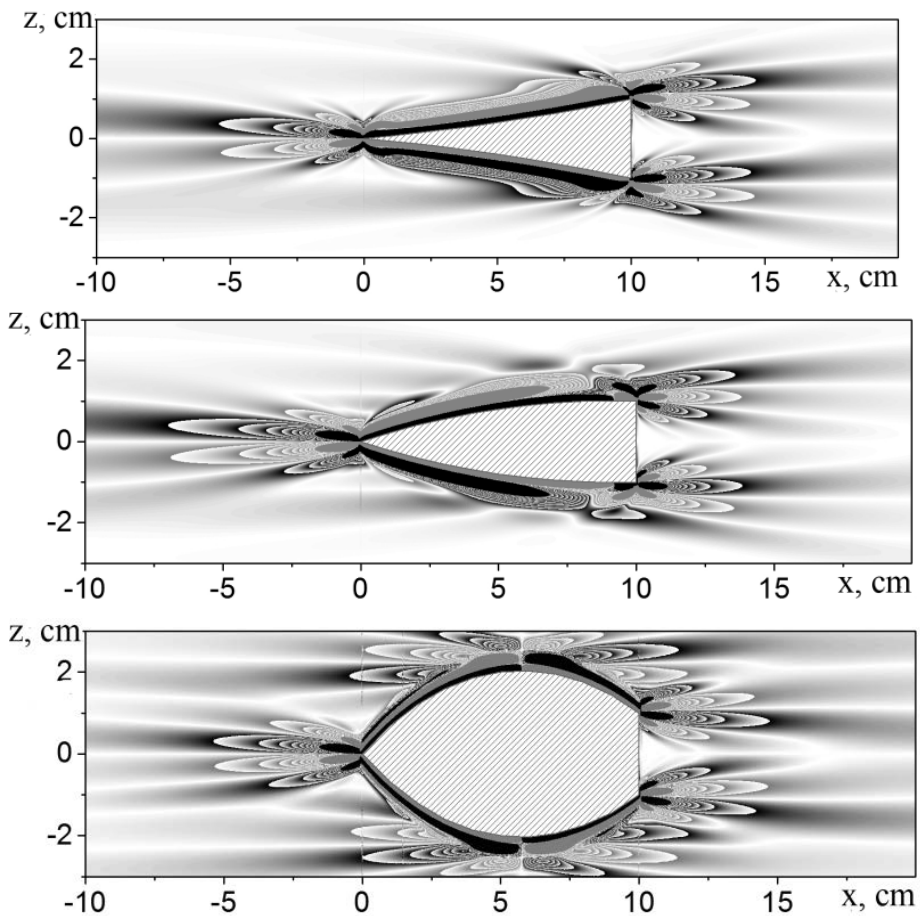

Fig. 1. Field of the horizontal component of salinity perturbation gradient $\partial s / \partial x$ on the wedge with the curved edges $\left(L=10 \mathrm{~cm}, h=2 \mathrm{~cm}, T_{b}=6.28 \mathrm{c}, \tau=t / T_{b}=16\right): a-\Delta_{R}=-0.1 \mathrm{~cm} ; b-\Delta_{R}$ $=0.3 \mathrm{~cm} ; c-\Delta_{R}=1.4 \mathrm{~cm}$

Field Structure of the horizontal component of salinity gradient perturbation essentially depends on the sign of the curvature of the lateral edges: the more oblique the tops at the base of the wedge, the more pronounced the visualized beams of alternating bands are (Fig. 1, a). At the same time the maximum values $|\partial s / \partial x|_{\max }$ weakly depend on the curvature of the wedge faces.

For a convex wedge (Fig. 1, $b$ ) with the angle between the base and the side close to $90^{\circ}$, the beam of fine structure elements spreads.

For the wedge with maximum height shifted relatively to the base, the pattern of flows is reconstructed (Fig. 1, $c$ ) and becomes similar to the one observed in the cylinder [8].

Impenetrable obstacle immersed in the fixed stably stratified fluid forms a complex system of flows, including the main thin jets from the adjacent compensation counterflows along the sloping sides (Fig. 2). When the obstacle is removed, the width of layers increases, and the maximum velocity drops.

In the distribution of the horizontal velocity component $v_{x}$ the main jet thickness of the adjacent flow increases along with a decrease of the local tangential tilt angle to the neutral buoyancy horizon. Accordingly, for the concave wedge the thickness is reduced from $0.8 \mathrm{~cm}$ nearby the sharp top to $0.2 \mathrm{~cm}$ near the base (Fig. 2, a), and as for the convex wedge, it, on the contrary, increases (Fig. 2, $b)$. 

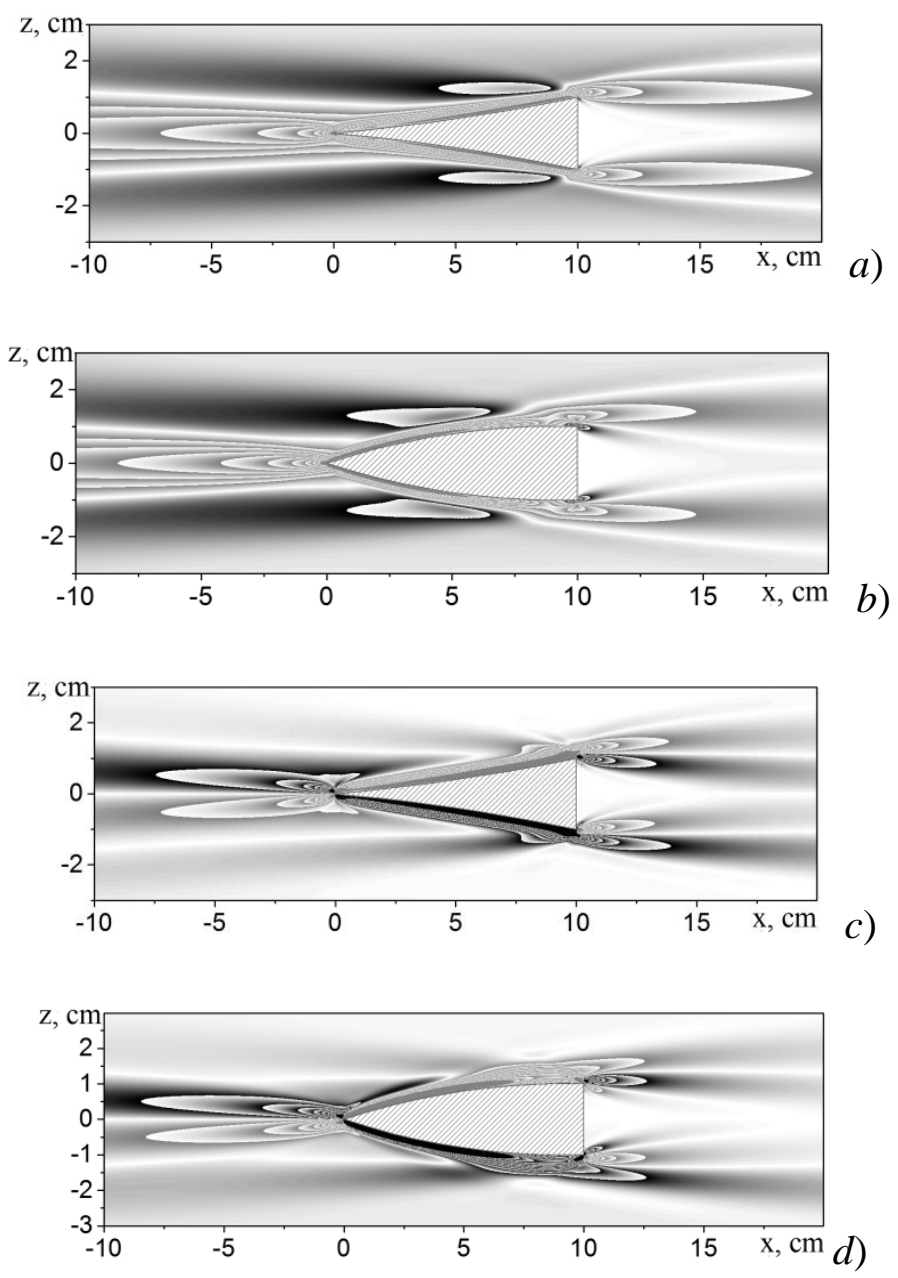

Fig. 2. Components of the flow velocity on the wedge $\left(L=10 \mathrm{~cm}, h=2 \mathrm{~cm}, T_{b}=6.28 \mathrm{~s}\right.$, $\left.\left.\tau=\tau / T_{b}=16\right): a, b\right)$ - longitudinal $\left.v_{x} ; c, d\right)$ - vertical $\left.\left.v_{z} ; a, c\right)-\Delta_{R}=-0.1 \mathrm{~cm} ; b, d\right)-\Delta_{R}=0.3 \mathrm{~cm}$

In the field of the vertical velocity component $v_{z}$ (Fig. 2, $c, d$ ) the main jets (layers having maximum velocity value) detach from the surface at the corner points at the wedge base and form a system of dissipative gravitational waves. There is an unperturbed area in the middle of the wedge base, as the vertical surface does not violate the diffusion impurity transfer.

Distributions of the flow velocity components near the wedge tops are shown in Fig. 3. Near the sharp top, the flow bifurcation point, there are precipitous changes in the vertical component of the velocity (curves 1 and 2 in Fig. 3, b). The values of the velocity components depend on the angle of wedge inclination edge to the $x$ axis.

Around the corner points of the wedge base the complex alternating structure is formed. There the vertical component of the velocity $v_{z}$ is comparable to the absolute value of the horizontal one $v_{x}$ (curves 3 and 4 in Fig. 3, b). 

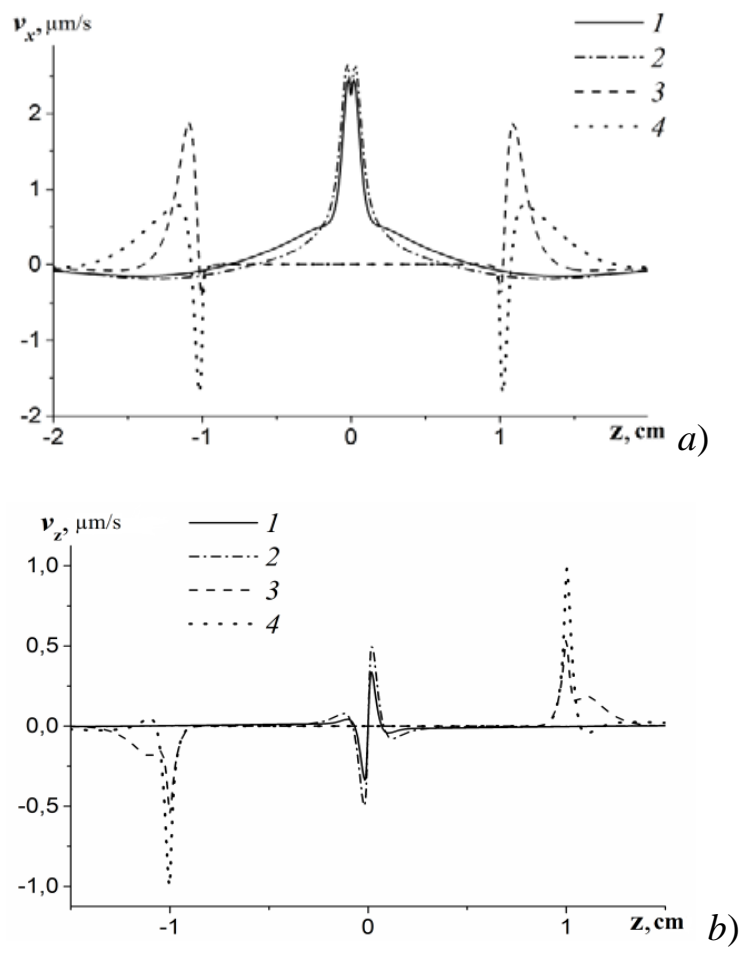

Fig. 3. The profiles of velocity component at top (curves 1 and 2) and lateral base (curves 3 and 4 ) of the wedge ( $\left.L=10 \mathrm{~cm}, h=2 \mathrm{~cm}, T_{b}=6.28 \mathrm{~s}, \tau=\tau / T_{b}=16\right): a$ ) - longitudinal $\left.v_{X} ; \sigma\right)$ - vertical $v_{z}$; curves $1,3-\Delta_{R}=-0.1 \mathrm{~cm} ; 2,4-\Delta_{R}=0.3 \mathrm{~cm}$

In the sharp top of the wedge, and also in the small flow along its lateral sides the pressure perturbation decreases (Fig. 4). Its value depends on the curvature of the sides. Thus, the thickness of the pressure deficit extended area on the concave wedge under $\Delta_{R}=-0.1 \mathrm{~cm}$ is about $3 \mathrm{~cm}$ (Fig. 4, a). It is significantly more than on the convex wedge: under $\Delta_{R}=0.3 \mathrm{~cm}$ the thickness of this area is $2.0 \mathrm{~cm}$, under $\Delta_{R}=1.4-1.5 \mathrm{~cm}$ (Fig. 4, c). For a convex wedge in Fig. 4, there is a deficit of pressure in all the tops, due to change of the edge inclination angle.

The pressure difference creates a horizontal component of an integral force $F_{x}$, pushing the horizontal wedge towards the top, which is consistent with the observations in $[11,12]$. The calculated values of $F_{x}$ are shown in Tab. 2 when $\tau=t / T_{b}=16$.

Table 2

Dependence of the Horizontal Component of the Composite Force

Acting on a Wedge and the Curvature of its Surfaces $\left(\tau=t / T_{b}=16\right)$

\begin{tabular}{c|ccccc}
\hline$\Delta_{R}, \mathrm{~cm}$ & -0.1 & 0.0 & 0.1 & 0.3 & 1.4 \\
$F_{x}, \mathrm{H}$ & $8.4 \cdot 10^{-6}$ & $8.3 \cdot 10^{-6}$ & $8.0 \cdot 10^{-6}$ & $-6.1 \cdot 10^{-6}$ & $-2.2 \cdot 10^{-6}$ \\
\hline
\end{tabular}



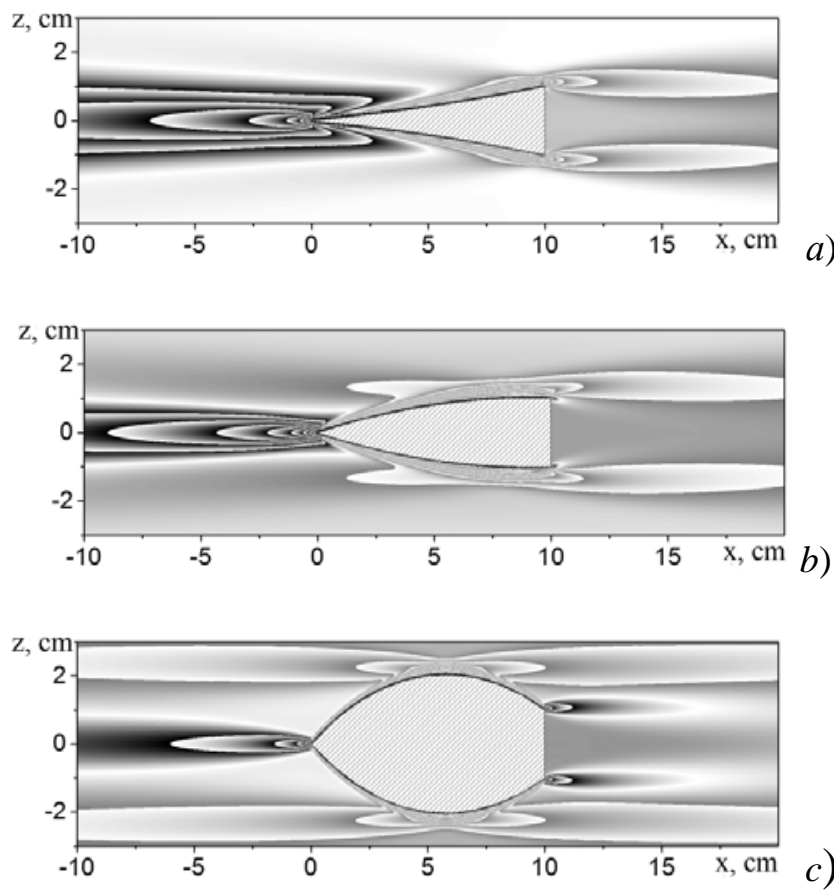

Fig. 4. Fields of pressure perturbations on the wedge ( $\left.L=10 \mathrm{~cm}, h=2 \mathrm{~cm}, T_{b}=6.28 \mathrm{~s}, \tau=16\right)$ : $\left.a-c\right)-$ $\Delta_{R}=-0.1 ; 0.3 ; 1.4 \mathrm{~cm}$

The vertical component of the composite force $F_{z}$, calculated separately for the top and bottom edges, is in excess of $F_{X}$ in two orders. Due to the uncollinearity, they compensate each other's action.

Conclusion. The mathematical model and the numerical implementation method permitting to study simultaneously all the elements of the internally multiscale stratified currents without additional hypotheses and links are developed on the basis of the fundamental system of equations. Criteria for the reconstruction and optimization of the grids are formulated based on the large-scale analysis of systems of equations. For the first time, the induced diffusion flows on a fixed symmetrical wedge with the curved edges are calculated and visualized using the supercomputer systems.

Influence both of the edge effects and the wedge lateral edge curvature upon the current structure is shown. In a stably stratified environment, the integral force inducing self-motion of a free wedge along the neutral buoyancy horizon towards the top is formed by pressure deficit in the adjacent jet streams. In accordance with the results of the experiments, the body in a stably stratified medium moves toward its top. The pressure perturbation value depends on the curvature of the lateral sides of the wedge.

Thin fluid jet streams, formed along each side of the wedge, generate the internal waves in the areas of convergence with the corner points. These waves form thin layers. 
The results of calculations of the induced diffusion flows can be used as initial conditions for solving problems of flow of the stratified marine environment past obstacles.

Acknowledgements. The computations were carried out in UniHUB web laboratory (www.unihub.ru) and visualized by supercomputer in CC MSU Lomonosov (www.parallel.ru). The research was conducted in A. Ishlinsky Institute for Problems in Mechanics RAS under the financial support of RFBR (Project No. 15-01-09235) and Department of Energy, Engineering, Mechanics and Control Processes, RAS (Program IV-4-12 Dynamics of formation and interaction of waves and vortices in continuous media).

\section{REFERENCES}

1. Prandtl', L., 1949, “Gidroaeromekhanika [Hydroaeromechanics]”, Moscow, Izd-vo inostr. lit., 488 p. (in Russian).

2. Phillips, O.M., 1970, “On flows induced by diffusion in a stably stratified fluid”, Deep-Sea Res., vol. 17, pp. 435-443.

3. Shapiro, A., Fedorovich, E., 2014, “A boundary-layer scaling for turbulent katabatic flow”, Bound.-Lay. Meteorol., vol. 153, iss. 1, pp. 1-17.

4. $\quad$ Oerlemans, J., Van Pelt, W.J.J., 2015, “A model study of Abrahamsenbreen, a surging glacier in northern Spitsbergen”, The Cryosph., vol. 9, no 2, pp. 767-779.

5. Zyryanov, V.N., Lapina, L.E., 2012, "Sklonovye techeniya $v$ moryakh, ozerakh vodokhranilishchakh, obuslovlennye diffuzionnymi effektami [Slope currents in the seas, lakes and reservoirs due to diffusion effects]”, Vodnye resursy, vol. 39, no. 3, pp. 292-303 (in Russian).

6. Garrett, C., MacCready, P. \& Rhines, P.B., 1993, "Boundary mixing and arrested Ekman layers: rotating, stratified flow near a sloping boundary”, Ann. Rev. Fluid Mech., vol. 25, pp. 291-323.

7. Gargett, A.E., 2003, "Differential diffusion: an oceanographic primer”, Progr. Oceanogr., vol. 56, pp. 559-570.

8. Linden, P.F., Weber, J.E., 1977, "The formation of layers in a double-diffusive system with a sloping boundary”, J. Fluid Mech., vol. 81, pp. 757-773.

9. $\quad$ Chashechkin, Yu.D., Zagumennyy, Ya.V., 2012, “Techeniya nepreryvno stratifitsirovannoy zhidkosti, indutsirovannye preryvaniem diffuzionnogo perenosa nepodvizhnoy plastinoy [Continuously stratified fluid flows induced by disruption of the diffusion transfer by fixed plate]”, Morskoy gidrofizicheskiy zhurnal, no. 5, pp. 3-22 (in Russian).

10. Zagumennyi, Ia.V., Chashechkin, Yu.D., 2013, “The structure of convective flows driven by density variations in a continuously stratified fluid”, Physica Scripta, vol. 155, article id. 014034.

11. Mercier, M.J., Ardekani, F.M. \& Allshouse, M.R. [et al.], 2014, "Self-propulsion of immersed object via natural convection”, Phys. Rev. Lett., vol. 112, no. 20, p. 204501(5).

12. Page, M.A., 2010, "Propelled by diffusion”, Nature Physics, vol. 6, pp. 486-487.

13. Landau, L.D., Lifshits, E.M., 1986, "Teoreticheskaya fizika. T. VI. Gidrodinamika [Theoretical physics. Vol. VI. Hydrodynamics]”, Moscow, Nauka, 736 p. (in Russian).

14. Chashechkin, Yu.D., 2014, "Differentsial'naya mekhanika zhidkostey: soglasovannye analiticheskie, chislennye $i$ laboratornye modeli stratifitsirovannykh techeniy [Differential fluid mechanics: coordinated analytical, numerical and laboratory model of stratified currents]”, Vestnik MGTU im. N.E. Baumana. Ser. Estestvennye nauki, no. 6, pp. 67-95 (in Russian).

15. Dimitrieva, N.F., Zagumennyy, Ya.V., 2014, “Chislennoe modelirovanie stratifitsirovannykh techeniy $s$ ispol'zovaniem OpenFOAM [Numerical modeling of stratified flows using OpenFOAM]”, Tr. ISP RAN, vol. 26, no. 5, pp. 187-200 (in Russian). 